\title{
Neuropatía periférica como manifestación unica de poliangeítis microscópica en la altura. Reporte de caso
}

\section{Peripheral neuropathy as a unique manifestation of microscopic polyangetis in high altitude. Case report}

Malena Roque-Poma ${ }^{1,2, a}$, Leslie Ruiz-Marmolejo ${ }^{1,2, a}$, María Rojas-Puertas ${ }^{1,2, a}$, Carlos Camargo-Espinoza ${ }^{1,3}$, Amilcar Tinoco-Solorzano ${ }^{1,2,4, \mathrm{~b}}$

\section{RESUMEN}

La poliangeítis microscópica, es una vasculitis de vasos pequeños cuya incidencia es de 4 por 1000000 de habitantes y que frecuentemente se presenta asociada a manifestaciones de glomerulonefrítis rápidamente progresiva y capilaritis pulmonar. Las manifestaciones neurológicas son menos frecuentes. El diagnostico se confirma con serología positiva para Anticuerpos Anticitoplasma de neutrófilos (ANCA) y especificidad para patrón de fluorescencia perinuclear (P-ANCA) y Mieloperoxidasa (MPO). El tratamiento idóneo son los glucocorticoides e inmunosupresores. Se presenta el caso de un paciente de 69 años con poliangeitis microscópica que debuta con neuropatía periférica como única manifestación clínica, presentación que se considera atípica. Se plantea que ante la presencia de una neuropatía periférica aislada, deben efectuarse estudios para confirmar o descartar la presencia de Poliangeitis Microscópica en base a la gran variabilidad de su sintomatología clínica y potenciales formas de tratamiento.

PALABRAS CLAVE: Poliangeítis microscópica, vasculitis, neuropatía, altitud.

\section{SUMMARY}

Microscopic polyangiitis is a small vessel vasculitis whose incidence is 4 per 1000000 inhabitants, frequently associated with manifestations of rapidly progressive glomerulonephritis and pulmonary capilaritis, and a less frequent neurological involvement.

The diagnosis is confirmed with a positive serology for antineutrophil cytoplasmic antibodies (ANCA) and specificity for fluorescence pattern perinuclear (P-ANCA) and myeloperoxidase (MPO). The ideal treatment is a combination of glucocorticoids and immunosuppressants. The case is presented of a 69-year-old patient with microscopic polyangiitis who debuts with peripheral neuropathy as the only manifestation, an atypical feature. The suggestion is made that in the presence of an isolated peripheral neuropathy, studies should be done to rule out the presence of Microscopic Polyangiitis, given its great variability in clinical presentation and potential treatments.

KEYWORDS: Microscopic polyangiitis, vasculitis, neuropathy, high altitude.

Facultad de Medicina Humana, Universidad Peruana Los Andes. Huancayo, Perú.

2 Sociedad Científica de Estudiantes de Medicina Los Andes, Universidad Peruana Los Andes. Huancayo,Perú.

3 Servicio de Medicina Interna, Hospital Nacional Daniel Alcides Carrión, Ministerio de Salud. Huancayo, Perú.

4 Servicio de Cuidados Intensivos e Intermedios, Hospital Nacional Ramiro Priale Priale, EsSALUD. Huancayo, Perú.

a Estudiante de Medicina Humana

b Médico especialista en Medicina Intensiva 


\section{INTRODUCCIÓN}

La neuropatía periférica (NP) incluye a todas las afecciones que resultan de la lesión del sistema nervioso periférico, la prevalencia en la población general es del 2,4\%. Sus manifestaciones incluyen síntomas sensoriales, autonómicos, motores $\mathrm{y} / \mathrm{o}$ dolor neuropático (1). La electromiografía (EMG) y el estudio de conducción nerviosa $(\mathrm{ECN})$ son herramientas útiles para confirmar el diagnóstico evidenciando pérdida axonal, desmielinización o ambas y definir la gravedad de la neuropatía. La biopsia del nervio sural está indicada en las neuropatías mediadas por inflamación, infección o infiltración. El tratamiento ideal es sintomático y de acuerdo con la etiología encontrada (2).

La Poliangeítis Microscópica (PAM) es una vasculitis necrotizante sistémica con escasos depósitos inmunes o sin ellos, que afecta a los vasos de pequeño calibre. Afecta al riñón como glomerulonefritis rápidamente progresiva en casi el $100 \%$ y al pulmón como hemorragia alveolar entre el 25 al 90\% (3), El pico de ocurrencia esta alrededor de 60 y 65 años y la relación hombre/mujer es de 6:1 (4). Su incidencia es de 3 a 4 pacientes por cada 1000000 de habitantes con una prevalencia anual de 1 a 3 pacientes por 1 millón de habitantes. Tiene una mortalidad cercana al $20 \%$ durante el primer año (5). La etiología de la PAM se ha asociado con factores genéticos, infecciosos y/o ambientales Los marcadores serológico ANCA positivo con especificidad P- ANCA y MPO acercan al diagnóstico de PAM. El etiquetado citoplasmático p-ANCA, con especificidad de MPO argumenta para PAM, esta especificidad característica facilita el diagnóstico diferencial con la vasculitis de ChurgStraus y en especial con la Granulomatosis de Wegener $(\mathrm{GW})$. El tratamiento por excelencia es la combinación de metilprednisolona con ciclofosfamida hasta inactivar la enfermedad, utilizando posteriormente corticoides para el mantenimiento (6).

La asociación entre la NP y PAM se da en un porcentaje de entre 11 al $52 \%$ en pacientes con afectación renal y/o pulmonar pudiendo en muy raras ocasiones ser la primera manifestación de la enfermedad $(7,8)$, Sin embargo, es muy infrecuente su presentación única. El 2011 se reportó un caso de un paciente de 43 años, que demuestra que si es posible encontrar NP como manifestación atípica de PAM (7). Nuestro objetivo fue presentar un caso de debut atipica única e inusual de neuropatía periférica en una enfermedad poco frecuente como la poliangeitis microscópica. Asimismo, deseamos sugerir el estudio con marcadores serológico para PAM en pacientes con NP. El presente caso clínico cumple con los criterios éticos, Para asegurar la confidencialidad del paciente se codifico la identidad, la historia clínica y sus anexos, al cual solo tuvo acceso el investigador principal, también se obtuvieron el consentimiento informado del paciente y el visto bueno del comité de ética institucional.

\section{Caso clínico}

Mujer de 69 años que ingresa al Hospital Nacional Daniel Alcides Carrión, ubicado en la ciudad de Huancayo a 3,350 msnm (Presión barométrica: 535 $\mathrm{mmHg}$ ), con antecedente de cáncer de útero hace 10 años, tratada con radioterapia e histerectomía. No refiere antecedente de diabetes mellitus, patología de tiroides. Ingresó con un mes de enfermedad, caracterizado por astenia, pérdida de fuerza en miembros inferiores, artromialgias, parestesias, pérdida ponderal de $10 \mathrm{Kg}$, deposiciones líquidas, palidez y sensación de alza térmica. En la exploración se evidencia: temperatura corporal $38^{\circ} \mathrm{C}$, frecuencia respiratoria 20 por minutos, frecuencia cardiaca 80 por minuto y presión arterial $100 / 80 \mathrm{mmHg}$ y palidez generalizada en las mucosas y tegumentos En el examen de los miembros inferiores se evidencia dolor (6/10) a la movilización de las articulaciones de las rodillas, hipotrofia muscular, debilidad muscular bilateral distal y asimétrica que permite movimientos contra la gravedad pero sin resistencia, reflejos osteotendinosos presentes, no ataxia.

Los análisis de laboratorio iniciales indicaron hemoglobina $12,4 \mathrm{~g} / \mathrm{dL}$, linfocitos $16,4 \%$, neutrófilos $77,6 \%$, glucosa $100 \mathrm{mg} / \mathrm{dL}$, creatinina $0,57 \mathrm{mg} / \mathrm{dL}$, urea $40 \mathrm{mg} / \mathrm{dL}$, proteinuria en orina de 24 horas 0,30 $\mathrm{g} / 24 \mathrm{~h}$ y un sedimento urinario con albumina $(++)$, gérmenes $(+++)$, nitritos positivos, piocitos 1-2 por campo y leucocitos 12-15 por campo. En el perfil de coagulación se evidencio tiempo de protrombina prolongado y factor RH positivo. En el perfil hepático, albumina en suero $2,53 \mathrm{~g} / \mathrm{dL}$ y proteínas totales $4,06 \mathrm{~g} /$ dL. TSH 2.37 mUI/L T4 10 ug/dL y T3 135 ng/dL.

Al no poder diagnosticar clínicamente la causa de la NP, ante la ausencia de alteraciones en el tono muscular, los reflejos y debido al rápido deterioro de la función motora de los miembros inferiores y superiores, se realizó una electromiografía. La electromiografía mostro mononeuritis multiple sensitiva motora de tipo axonal con predomio derecho, aguda a subaguda 
con signos de reinervación. La punción lumbar sin evidencia de celularidad no compatible con Síndrome Guillain Barre. En la biopsia del nervio sural, se concluyó leve edema endoneural y ocasionales células de infiltrado inflamatorio linfocitario perineural asociado de degeneración axonal.

El diagnóstico de la Poliangeitis incluyo: a) Serología para factor reumatológico de $525,8 \mathrm{mg} / \mathrm{dL}$ $(<60 \mathrm{u} / \mathrm{ml}$ negativo), anticuerpos anticitoplasma de neutrófilos (ANCA) positivo, ANCA en etanol con patrón perinuclear, proteína mieloperoxidasa (MPO) de 32,7 $(<0,9 \mathrm{U} / \mathrm{ml}$ negativo), Proteína $\mathrm{C}$ reactiva cualitativo $(+++)$, las determinaciones de anticuerpos antinucleares (ANA) negativo; proteinasa 3 (PR3) de $1,9(<3,5 \mathrm{U} / \mathrm{ml}$ negativo) y péptido cítrico citrulinado de 11,2 ( 0-20 U/ml negativo) ambos dentro de los valores normales. b) Estudios de imágenes reportaron: Radiografía de tórax sin hallazgos significativos, TAC de Abdomen y Pelvis con evidencia de leve hidronefrosis derecha aparentemente condicionada por engrosamiento de las paredes renales, y dilatación de la vía biliar. c) Biopsia del nervio sural relacionado a degeneración axonal.

Tras la confirmación del diagnóstico de Poliangeitis Microscópica por medio de la clínica sugestivo de NP, serología positiva para PAM (ANCA, P-ANCA Y MPO), EMG compatible con neuropatía periférica motora de tipo axonal y biopsia del nervio sural sugestivo de patología inflamatoria axonal, se inició tratamiento con metilprednisolona de $500 \mathrm{mg}$ vía endovenosa por 3 días, posteriormente se pasó a prednisona de $1 \mathrm{mg} / \mathrm{kg} /$ día vía oral cada 12 horas por 7 días. Actualmente paciente se encuentra estable, con mejoría clínica notable, en seguimiento por consultorio externo de reumatología, neurología y medicina interna.

\section{DISCUSIÓN}

En nuestro caso clínico, se llegó el diagnóstico de PAM por la presencia de síntomas de neuropatía periférica; tales como disminución de la masa y fuerza muscular en extremidades asociados a parestesias en miembros inferiores. La presencia de marcadores serológicos ANCA positivo con especificidad para P-ANCA y MPO, la EMG y una biopsia del nervio sural confirmaron el diagnóstico de poliangeítis microscópica.

En este caso la paciente no presentaba clínica ni exámenes auxiliares de hemorragia alveolar, y a pesar de presentar proteinuria compatible con glomerulonefrítis rápidamente progresiva (afectaciones debut típicas de PAM), los síntomas debut más intensos que obligaron su estudio fueron neuropáticos, manifestaciones poco comunes que retrasaron el diagnóstico y el tratamiento. La PAM comparte similitudes con la vasculitis de Churg-Straus y la Granulomatosis de Wegener (GW), sin embargo, existen diferencias decisivas; la GW presenta afectación de vías respiratorias superiores, formación de granulomas y ANCA positivo pero con especificidad para c-ANCA y anti-proteinasa 3 (PR3) (8-10). La vasculitis de Churg-Straus, a pesar de compartir el mismo resultado en laboratorio con PAM, presenta el antecedente de asma (2), dato que la paciente no presentaba por lo cual, se excluyó este diagnóstico. Valores dentro de parámetros normales de glicemia y hormonas tiroideas descartaron diabetes mellitus y enfermedad tiroidea. Ausencia de anemia, reflejos osteotendinosos normales y ataxia alejaron la posibilidad de déficit de vitamina B12.

La electromiografía (EMG) y la velocidad de conducción nerviosa (VCN) realizada al paciente confirmó la neuropatía periférica sensitiva motora de tipo axonal $2(2,7,9)$. La biopsia del nervio sural mostro hallazgos de edema y reacción inflamatoria neural. (2).

El inicio del tratamiento no debe ser retrasado hasta que se haya realizado el diagnóstico de la enfermedad, dado que el pronóstico de esta depende de la rapidez de instauración de la terapia (7). la paciente recibió tratamiento inmediato con metilprednisolona, sin embargo, no llego a recibir el tratamiento ideal debido a la ausencia de ciclofosfamida en el centro hospitalario. El uso de plasmaféresis se inicia ante la presencia de falla renal y/o hemorragia pulmonar (6), siendo innecesario en este caso.

Se describe una presentación inusual tal como la neuropatía periférica en una enfermedad poco frecuente como la Poliangeitis Microscópica, Los marcadores serológicos confirmaron el diagnostico, motivo por el cual planteamos la realización de estos estudios ante la presencia de casos de neuropatía periférica de tipo subaguda con EMG axonal, y cuando las causas más frecuentes se hayan descartado.

\section{Correspondencia:}

\section{Amilcar Tinoco-Solorzano}

Av. Independencia 266 El Tambo, Huancayo, Junín, Perú.

Correo electónico: amilcartinoco@gmail.com

Teléfono: 51952991010 
Fuentes de financiamiento: Este artículo ha sido financiado por los autores.

Conflictos de interés: Los autores declaran no tener ningún conflicto de interés.

\section{REFERENCIAS BIBLIOGRÁFICAS}

1. Callaghan B, Price R, Chen K, Feldman E. Peripheral neuropathy: The importance of rare subtypes. JAMA Neurol. 2015; 72(12): 1510-1518.

2. Watson J, Dyck J. Peripheral neuropathy: A practical approach to diagnosis and symptom management. Mayo Clinic. 2015;90(7):940-951

3. Sinico RA, Di Toma L, Radice A. Renal involvement in antineutrophil cytoplasmic autoantibody associated vasculitis. Autoimmun Rev. 2013;12(4):477-82.

4. Parra F, Echeverri J, Polo J, Carrillo J. Presentación atípica de la Poliangetis microscópica con compromiso renal, pulmonar, dermatológico y del sistema nervioso central. Repertorio de Medicina y Cirugia,2018;27(2): 119-123.
5. Santiago D. Enfrentamiento de las vasculitis primarias. Clinical aspects of primary vasculitis. Rev Med Clin Condes. 2012; 23(4): 403-411.

6. Trénor L, Martínez C, Pallardó C. Vasculitis sistémicas asociadas a ANCA- Granulomatosis de Wegener y Poliangeítis Microscópica. Alzira. 2008; 14: 263-276

7. Tejero M, Guerrero A, Hernández E, et al. Neuropatía periférica como forma de presentación de poliangeitis microscópica. Neurologia. 2010; 26(5):257-318. DOI: 10.1016/j.nrl.2010.09.018

8. Chung SA, Seo P. Microscopic polyangiitis. Rheum Dis Clin North Am. 2010;36(3):545-58.

9. Villiger P, Guillevin L. Microscopic Polyangiitis: Clinical presentation. Autoimmunity Reviews. 2010;9:81-819.

10. Wilke L, Prince-Fiocco M, Fiocco GP. Microscopic polyangiitis: a large single-center series. J Clin Rheumatol. 2014;20(4):179-82.

Recibido: 04/05/2020 Aceptado: 16/06/2020 